\title{
Validation of the Clavien-Dindo grading system of complications for microsurgical treatment of unruptured intracranial aneurysms
}

\author{
Martina Sebök, MD, 1,2 Patricia Blum, BMed, ${ }^{1,2}$ Johannes Sarnthein, PhD, 1,2 \\ Jorn Fierstra, MD, PhD, ${ }^{1,2}$ Menno R. Germans, MD, PhD, 1,2 Carlo Serra, MD, 1,2 \\ Niklaus Krayenbühl, MD,1,2 Luca Regli, MD, 1,2 and Giuseppe Esposito, MD, PhD1,2 \\ ${ }^{1}$ Department of Neurosurgery, University Hospital Zurich, University of Zurich, Zurich, Switzerland; and ${ }^{2}$ Clinical Neuroscience \\ Center, University Hospital Zurich, Zurich, Switzerland
}

\begin{abstract}
OBJECTIVE Microsurgery plays an essential role in managing unruptured intracranial aneurysms (UIAs). The ClavienDindo classification is a therapy-oriented grading system that rates any deviation from the normal postoperative course in five grades. In this study, the authors aimed to test the applicability of the Clavien-Dindo grade (CDG) in patients who underwent microsurgical treatment of UIAs.
\end{abstract}

METHODS The records of patients who underwent microsurgery for UIAs (January 2013-November 2018) were retrieved from a prospective database. Complications at discharge and at short-term follow-up ( 3 months) were rated according to the Clavien-Dindo system. Patient outcomes were graded using the modified Rankin Scale (mRS) and the National Institutes of Health Stroke Scale (NIHSS). A descriptive statistic was used for data analysis.

RESULTS Overall, 156 patients underwent 157 surgeries for 201 UIAs (size range 4-42 mm). Thirty-nine patients (25\%) had complex UIAs. An adverse event (CDG $\geq I$ ) occurred in 21 patients (13.5\%) by the time of discharge. Among these, 10 patients $(6.4 \%)$ presented with a new neurological deficit. Significant correlations existed between a $C D G \geq I$ and an increase in $\mathrm{mRS}$ and $\mathrm{NIHSS}$ scores $(p<0.001)$. Patients treated for complex aneurysms had a significantly higher risk of developing new neurological deficits ( $20.5 \%$ vs $1.7 \%, p=0.007$ ). At the 3 -month follow-up, a CDG $\geq$ I was registered in 16 patients (10.3\%); none presented with a new neurological deficit. A CDG $\geq$ I was associated with a longer hospital length of stay (LOS) (no complication vs $C D G \geq 1,6.2 \pm 3.5$ days vs $9.3 \pm 7.7$ days, $p=0.02$ ).

CONCLUSIONS The CDG was applicable to patients who received microsurgery of UIAs. A significant correlation existed between CDG and outcome scales, as well as LOS. The aneurysm complexity was significantly associated with a higher risk for new neurological deficit.

https://thejns.org/doi/abs/10.3171/2021.8.FOCUS20892

KEYWORDS unruptured intracranial aneurysm; complications; Clavien-Dindo grade; microneurosurgery; clipping

$\mathrm{U}$ NRUPTURED intracranial aneurysms (UIAs) are incidentally detected at an increasing rate, due to the higher number of noninvasive neuroimaging studies performed..$^{1-4}$ Despite the expansion of neurointerventional techniques, microsurgery still plays an important role in managing UIAs.

It is important to report and analyze treatment-related complications completely and objectively, ideally in a prospective manner, to improve the outcome for patients. The
Clavien-Dindo classification is a therapy-oriented grading system developed to categorize adverse events depending on the type of treatment required for a given complication. The Clavien-Dindo grade (CDG) rates any deviation from the normal and expected postsurgical course in five grades and considers not only surgery-related but also anesthesiarelated and hospital stay-related complications. ${ }^{5,14} \mathrm{CDGs}$ can either remain stable or worsen (increase) over time; in fact, any new complication occurring during the postop-

ABBREVIATIONS CDG = Clavien-Dindo grade; ICU = intensive care unit; LOS = length of stay; $M C A=$ middle cerebral artery; $m R S=$ modified Rankin Scale; NIHSS = National Institutes of Health Stroke Scale; UIA = unruptured intracranial aneurysm.

SUBMITTED October 21, 2020. ACCEPTED August 20, 2021.

INCLUDE WHEN CITING DOI: 10.3171/2021.8.FOCUS20892. 
erative course is considered and then graded. Therefore, CDGs cannot improve (decrease) over time. This contrasts with the outcome scales, which can improve and worsen over time depending on the patient's clinical condition.

This study aims to test the applicability of the CDG in a well-defined neurosurgical patient population who underwent microsurgical treatment of noncomplex and complex UIAs. Moreover, we studied the correlation of the CDG with neurological outcome scores as well as the impact on the hospital length of stay (LOS).

\section{Methods}

\section{Patient Selection}

All patients who underwent microsurgical treatment of UIAs at the Department of Neurosurgery, University Hospital Zurich, Switzerland, between January 2013 and November 2018 were included in this study. The indication and type of treatment (endovascular vs microsurgical) of patients with UIAs were discussed at the weekly multidisciplinary cerebrovascular board. Patients with both noncomplex and complex UIAs were included in the analysis. Aneurysm complexity is related to at least one of the following features: 1) size $\geq 2.5 \mathrm{~cm}$; 2) anatomical location (vertebral, basilar, or paraclinoidal); 3) involvement of critical perforating or branch vessels; 4) previous treatment (endovascular or surgical); 5) dissecting, fusiform, saccular lesions with a very broad neck; 6) intraluminal thrombosis; and 7) atherosclerotic plaques and calcifications of the aneurysm wall and/or neck..$^{3,6-12}$

All patients underwent appropriate preoperative neuroimaging to precisely understand the angioanatomy with MRA, CTA, or DSA, including 3D reconstruction. Specialized vascular neurosurgeons performed all surgeries with the standard use of intraoperative neuromonitoring. Postoperative neuroimaging (CTA and/or DSA) was performed within the first 72 hours after surgery. A shortterm clinical follow-up within 3 months after surgery was performed in all patients.

\section{Assessment Tools for Clinical Outcome}

Patient outcomes were graded according to the modified Rankin Scale (mRS) and the National Institutes of Health Stroke Scale (NIHSS). The LOS was recorded for every patient.

\section{Complication Grading System}

Complications were analyzed and their severity was graded according to the CDG. ${ }^{13,14}$ The $\mathrm{CDG}$ registers any deviation of the normal postoperative course and rates these deviations in five grades: $\mathrm{CDG}$ I = any deviation from the normal postoperative course; $\mathrm{CDG}$ II $=$ requiring pharmacological treatment; CDG IIIa = requiring surgical intervention without general anesthesia; CDG IIIb = requiring surgical intervention with general anesthesia; $\mathrm{CDG}$ $\mathrm{IVa}=\mathrm{a}$ life-threatening complication, intensive care unit $(\mathrm{ICU})$, and single-organ dysfunction; $\mathrm{CDG}$ IVb $=$ a lifethreatening complication, ICU, and multiorgan dysfunction; and $\mathrm{CDG} \mathrm{V}=$ death within 30 days. ${ }^{14} \mathrm{CDG}$ grades also include minor reversible events such as urinary tract infections, pneumonia, or mild allergic reactions. . $^{5,13,14}$
In the CDG classification of $2004,{ }^{14}$ a suffix "d" was added to indicate any disabling complication persisting at the time of discharge or at the follow-up which compromises the patient's health and is directly related to the treatment. A frail health status after a long course of the disease does not qualify for the label "d."13

In our practice, the suffix "d" indicates any (disabling and nondisabling) new neurological deficit after a neurosurgical procedure that is still present at discharge. Consequently, to qualify for the suffix "d" at clinical followup, the patient had to have developed a new neurological deficit that was not present on admission or at discharge.

\section{Clinical Data Acquisition}

Patient data and outcomes were prospectively collected in an institutional patient registry, which was approved upfront by the local ethics review board (Kantonale Ethikkommission) and registered at clinicaltrials.gov (identifier no. NCT01628406). Since the systematic registration of suffix "d" was introduced to our registry in September 2017, the "d" values for patients who underwent microsurgical UIA treatment before were recorded retrospectively. Patient consent was waived because of the observational nature of the study.

All physicians were trained and certified to grade complications and clinical scores precisely for the correct use. The operating surgeon validated all discharge reports and all reported complications. Each new neurological deficit was further discussed at the monthly morbidity and mortality meeting. For ease of handling, we report only the highest CDG of each patient.

\section{Statistical Analysis}

All analyses were carried out in R version 3.6.1 (The R Foundation for Statistical Computing). ${ }^{15}$ All normally distributed continuous variables are reported as mean \pm standard deviation. Categorical, ordinal variables are presented as median (IQR), whereas dichotomous variables are shown as frequency. Significance was accepted at a $\mathrm{p}$ $<0.05$.

For the complete patient cohort, the association between CDG at any time point and outcome (mRS and NIHSS) or LOS was assessed using the Spearman's rank correlation and presented with rho effect sizes and $p$ values. Rho was interpreted as follows: $0.0 \leq$ rho $\leq 0.2$ indicates none to very slight correlation; $0.2 \leq$ rho $\leq 0.5$ indicates mild to moderate correlation; $0.5 \leq$ rho $\leq 0.8$ indicates clear correlation; and $0.8 \leq$ rho $\leq 1.0$ indicates high to perfect correlation. The complete patient cohort was divided into complication $(\mathrm{CDG} \geq \mathrm{I})$ and no-complication groups to assess the impact of the CDG on the outcome, and the outcome scales between these groups were compared using the Wilcoxon rank-sum test. The complication rate between patients with complex and noncomplex aneurysms and between patients with single and multiple aneurysms was assessed using the chi-square test.

\section{Results}

Overall, 156 patients underwent 157 surgical treatments of 201 UIAs (size range $4-42 \mathrm{~mm}$ ), including 37 patients 
TABLE 1. Baseline characteristics

\begin{tabular}{lc}
\hline & Value \\
\hline Female sex & $115(73.7)$ \\
\hline Mean age, yrs & $51.7 \pm 11.0$ \\
\hline Previous SAH & $35(22.4)$ \\
\hline$\geq 1$ complex aneurysm & $39(25)$ \\
\hline Multiple aneurysms & $37(24)$ \\
\hline No. of treated aneurysms & 201 \\
\hline Aneurysm treatment procedure & $198(98.5)$ \\
\hline Clipping & $191(95.0)$ \\
\hline Trapping w/ bypass & $7(3.5)$ \\
\hline Preop outcome scales & $7(4.5)$ \\
\hline mRS $\geq 3$ & $3(1.9)$ \\
\hline NIHSS $\geq 5$ & $12(7.7)$ \\
\hline Discharge outcome scales & $4(2.6)$ \\
\hline mRS $\geq 3$ & \\
\hline NIHSS $\geq 5$ & $6(3.8)$ \\
\hline Follow-up outcome scales & $2(1.3)$ \\
\hline mRS $\geq 3$ &
\end{tabular}

SAH = subarachnoid hemorrhage.

Values represent the number of patients (\%) or mean \pm SD.

(24\%) who had more than 1 UIA treated in the same surgery. Thirty-nine patients (25\%) were treated for at least 1 complex aneurysm, 4 of whom underwent treatment for 2 complex aneurysms, totaling 43 complex aneurysms from 201 cumulative UIAs $(21 \%)$ (Table 1). Thirteen patients (8.3\%) had received previous treatment. Flow-preservation bypass surgery was performed in 7 patients $(4.5 \%)$. Preoperatively, 7 patients $(4.5 \%)$ presented with an mRS score $\geq 3$ and 3 patients (1.9\%) with an NIHSS score $\geq$ 5. On postoperative imaging, complete aneurysm occlusion was achieved in all but 3 UIAs (98.5\%). One complex middle cerebral artery (MCA) aneurysm was not treated because of dropping of intraoperative somatosensory and motor evoked potentials after multiple clip positioning attempts. ${ }^{16}$ Another complex recurrent MCA aneurysm (treated by clipping and wrapping 21 years previously) was explored. It was intraoperatively considered not treatable because of severe perianeurysmal fibrosis impeding correct aneurysm dissection and proper understanding of the aneurysm angioanatomy. The third complex, giant MCA aneurysm was not entirely treated due to the failure of a second flow-preservation bypass. The mortality rate was $0 \%$.

\section{CDG at Discharge}

At discharge, any deviation of the normal postoperative course $(\mathrm{CDG} \geq \mathrm{I})$ was recorded in 21 patients $(13.5 \%)$, with a median CDG of II (IQR 2) (Table 2). Of these complications, those found in 10 patients $(6.4 \%)$ were additionally graded with a "d," indicating new neurological deficit present at discharge.

Poor neurological outcome (mRS score $\geq 3$ ) was re-
TABLE 2. List of complications at discharge and the 3-month follow-up according to CDG classification

\begin{tabular}{|c|c|c|c|}
\hline \multirow[b]{2}{*}{ CDG } & \multirow[b]{2}{*}{ Definition } & \multicolumn{2}{|c|}{ No. of Patients (\%) } \\
\hline & & $\begin{array}{l}\text { Discharge } \\
(n=156)\end{array}$ & $\begin{array}{c}\text { 3-Mo } \\
\text { Follow-Up } \\
(n=156)\end{array}$ \\
\hline & No complications, n (\%) & $135(86.6)$ & $140(89.7)$ \\
\hline \multirow{7}{*}{ I } & $\begin{array}{l}\text { Any deviation from the normal post- } \\
\text { op course, } n(\%)\end{array}$ & $8(5.1)$ & $1(0.6)$ \\
\hline & $\begin{array}{l}\text { New ischemia \& neurological } \\
\text { symptoms }{ }^{*}\end{array}$ & $4 d$ & \\
\hline & $\begin{array}{l}\text { Intraparenchymal hemorrhage w/ } \\
\text { new neurological symptoms }\end{array}$ & $1 \mathrm{~d}$ & \\
\hline & Amaurosis & $1 \mathrm{~d}$ & \\
\hline & Paresis of oculomotor nerve & $1 \mathrm{~d}$ & \\
\hline & latrogenic pneumothorax & 1 & \\
\hline & Wound healing complication & & 1 \\
\hline \multirow{8}{*}{ II } & $\begin{array}{l}\text { Requiring pharmacological treat- } \\
\text { ment, } n(\%)\end{array}$ & $9(5.8)$ & $4(2.6)$ \\
\hline & New visual field deficits & $1 d$ & \\
\hline & Epileptic seizure & 2 & 1 \\
\hline & Pneumonia & 2 & \\
\hline & Urinary tract infections & 2 & \\
\hline & Prolonged delirium & 2 & \\
\hline & Venous sinus thrombosis & & 1 \\
\hline & Wound healing complication & & 2 \\
\hline \multirow{7}{*}{ III } & $\begin{array}{l}\text { Illa: Requiring surgical intervention } \\
\text { w/o general anesthesia, } n(\%)\end{array}$ & $0(0)$ & $0(0)$ \\
\hline & $\begin{array}{l}\text { Illb: Requiring surgical intervention } \\
\text { w/ general anesthesia, } n(\%)\end{array}$ & $3(1.9)$ & $11(7.1)$ \\
\hline & $\begin{array}{l}\text { New ischemia \& neurological } \\
\text { symptoms }\end{array}$ & $1 d$ & \\
\hline & Acute epidural hematoma & 1 & \\
\hline & Bilat subdural hygroma & 1 & \\
\hline & Chronic subdural hematoma & & 2 \\
\hline & Wound healing complication & & 9 \\
\hline \multirow{3}{*}{ IV } & $\begin{array}{l}\text { IVa: Life-threatening complication, } \\
\text { ICU, single-organ dysfunction, } \mathrm{n}(\%)\end{array}$ & $1(0.6)$ & $0(0)$ \\
\hline & $\begin{array}{l}\text { New ischemia \& neurological } \\
\text { symptoms§ }\end{array}$ & $1 d$ & \\
\hline & $\begin{array}{l}\text { IVb: Life-threatening complication, } \\
\text { ICU, multiorgan dysfunction, } \mathrm{n}(\%)\end{array}$ & $0(0)$ & $0(0)$ \\
\hline V & Death w/in 90 days, $\mathrm{n}(\%)$ & $0(0)$ & $0(0)$ \\
\hline \multicolumn{2}{|c|}{ Total no. of complications, n (\%) } & $21(13.5)$ & $16(10.3)$ \\
\hline \multicolumn{2}{|c|}{ Total no. of new neurological deficits, n (\%) } & $10(6.4)$ & $0(0)$ \\
\hline \multicolumn{4}{|c|}{$\begin{array}{l}d=\text { new neurological deficit. } \\
\text { * Due to perianeurysmal perforators. } \\
\dagger \text { Due to a reperfusion hemorrhage after a high-flow bypass. } \\
\ddagger \text { Due to perianeurysmal perforators and required clip repositioning. } \\
\S \text { Due to severe spasm of the arterial interposition graft. }\end{array}$} \\
\hline
\end{tabular}




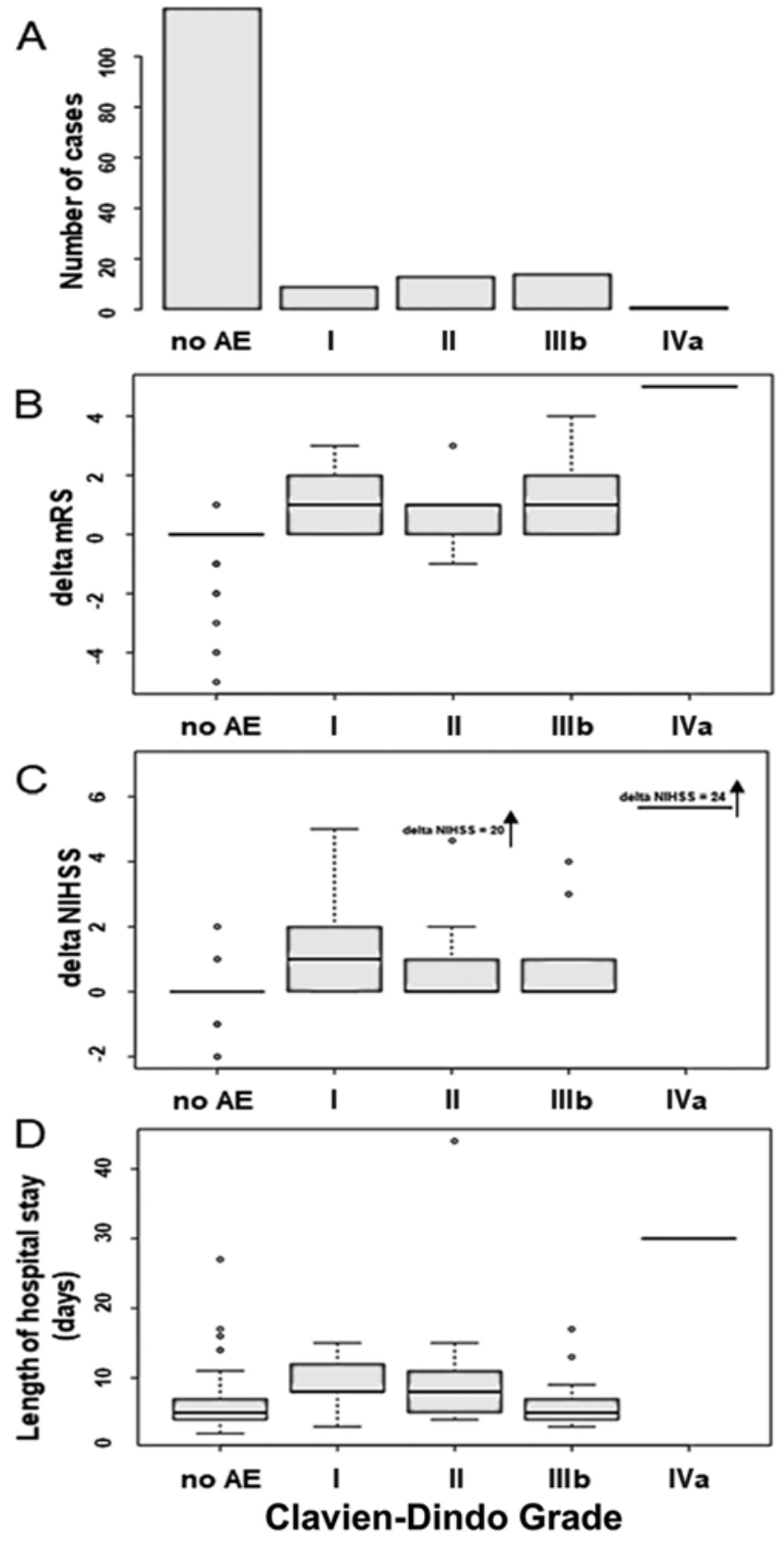

FIG. 1. Correlation between complications (CDG) occurring at any time point (discharge or follow-up) and outcome, as well as hospital LOS. A: Bar graph showing the distribution of the CDG of the 37 complications registered up to 3 months after discharge. B: Boxplot showing the correlation between CDG and change in (delta) $\mathrm{mRS}$ (rho $=0.61, p$ $<0.001$ ). C: Boxplot showing the correlation between CDG and delta NIHSS (rho $=0.42, p<0.001$ ). The delta NIHSS values of two outliers with high values due to scaling reasons were removed and then manually registered. D: Boxplot showing the correlation between CDG and hospital LOS (rho $=0.23, p=0.004)$. Delta mRS and delta NIHSS values have been calculated between the time point of the complication occurrence and the time point of the last well-being status, as follows. For complications registered at discharge, the delta values have been calculated between discharge mRS/NIHSS and admission mRS/ NIHSS; for complications registered at the 3-month follow-up, the delta values have been calculated between follow-up and discharge mRS/ NIHSS. FIG. 1. (continued) $\rightarrow$
FIG. 1. Among the 9 patients classified as CDG I, 7 patients experienced a new neurological deficit resulting in higher mRS and NIHSS scores. Since some of the neurological complications did not demand sophisticated (surgical or medical) treatments, they were graded low on the CDG scale, although they may be associated with worse patient outcomes. Therefore, we used the original CDG with an adaptation for neurosurgical patients, by using " $d$ " as an additional marker for any new neurological deficit after a neurosurgical procedure that is still present at discharge or follow-up. $\mathrm{AE}=$ adverse event.

corded in 12 patients $(7.7 \%)$ at discharge. Of these patients, $5(42 \%)$ had complex aneurysms and $3(25 \%)$ had presented with an mRS score $\geq 3$ preoperatively. Four patients presented with an NIHSS score $\geq 5$, and in 3 of those, an NIHSS score $\geq 5$ had been recorded preoperatively (Table 1).

\section{CDG at Follow-Up}

At the 3-month follow-up, a new complication since discharge, recorded as a $C D G \geq \mathrm{I}$, occurred in 16 patients (10.3\%) with a median CDG of IIIb (IQR 2); 12 patients had wound infections, 2 patients had chronic subdural hematomas, 1 patient had new epilepsy, and 1 patient had sinus vein thrombosis (Table 2). None of these patients experienced new neurological deficits. From 12 patients with an mRS score $\geq 3$ at discharge, 6 patients improved and presented with an mRS score $\leq 2$ at the follow-up. Two patients presented with an NIHSS score $\geq 5$, and both were recorded with an NIHSS score $\geq 5$ at discharge (Table 1).

\section{Correlation Between CDG and Outcome Scores}

Concerning the correlation between the CDG and the outcome scoring systems at discharge or follow-up, the study found a statistically significant association. In fact, the mRS showed a clear statistical correlation with rho = 0.61 and $p<0.001$. The NIHSS was moderately correlated with rho $=0.42$ and $p<0.001$ (Fig. 1).

\section{Difference in Complication Rate Between Single, Multiple, Complex, and Noncomplex Aneurysms}

Comparing patients treated for a single aneurysm (119, $76 \%$ ) and patients treated for multiple aneurysms (37, $24 \%$ ), we did not find a significant difference in the complication rate (single aneurysms 29 [24.4\%] vs multiple aneurysms 8 [21.6\%], $\mathrm{p}=0.73)$. For both groups, the median CDG was III (IQR 2 for patients with a single aneurysm and IQR 1 for patients with multiple aneurysms). No significant difference in the rate of new neurological deficits was seen between groups (single aneurysms 9 [7.6\%] vs multiple aneurysms 1 [2.7\%], $\mathrm{p}=0.18$ ).

Comparing patients treated for complex aneurysms (39, $25 \%)$ and patients treated for noncomplex aneurysms (117, $75 \%$ ), we found a statistical trend toward a higher complication rate in patients with complex aneurysms (complex aneurysms 14 [36\%] vs noncomplex aneurysms 23 [20\%], $\mathrm{p}=0.06$ ). Regarding the occurrence of a new neurological deficit, patients treated for complex aneurysms had a significantly higher risk of developing new neurological deficit compared with patients treated for noncomplex aneurysms $(8[20.5 \%]$ vs $2[1.7 \%], p=0.007)$. 


\section{Influence of Complications on Hospital LOS}

All patients entered the hospital a day before the planned surgery and stayed in the hospital a mean of 6.4 \pm 4.8 days. The median hospital LOS was 5 days (IQR 4 days [range 2-44 days]).

The occurrence of complications was associated with a longer hospital LOS $(9.4 \pm 7.8$ days vs $6.2 \pm 3.5$ days, $\mathrm{p}$ $=0.02)$. LOS was mildly associated with a higher CDG (rho $=0.23, p=0.004)$ (Fig. 1). The LOS was significantly longer if patients experienced a new neurological deficit compared with patients who had complications but no new neurological deficit $(17.3 \pm 11.3$ days vs $6.5 \pm 2.9$ days, $\mathrm{p}$ $=0.01$ ).

\section{Discussion}

\section{Feasibility of CDG to Classify Complications in UIA Surgery}

Our data show a statistically significant correlation between CDG and both outcome grading systems (mRS and NIHSS), which means that patients with higher CDGs at discharge presented with higher mRS and NIHSS scores. In our cohort, by the time of discharge, any adverse event during the postoperative course (CDG $\geq \mathrm{I}$ ) had occurred in $13.5 \%$ of patients and any new neurological deficit had occurred in $6.4 \%$ of patients, with an overall mortality rate of $0 \%$. Other series about microsurgical management of UIAs have reported disabling morbidity rates ranging between $3.4 \%$ and $10.9 \% .^{17-19}$

Kotowski et al. ${ }^{20}$ performed a systematic review and meta-analysis of 60 studies including 9845 patients with 10,845 aneurysms and found an overall morbidity rate of $6 \%$ to $7 \%$. Their definition of a complication only included neurological symptoms, not any deviation from the normal postoperative course, as is the case in the current study.

\section{Aneurysm Complexity and New Neurological Deficits}

It is essential to mention that in this cohort, 39 patients (25\%) were treated for a complex UIA. Flow-preservation bypass surgery was performed in 7 patients $(4.5 \%)$, and 13 patients $(8.3 \%)$ had undergone a previous treatment attempt. Treatment of complex aneurysms often requires the use of advanced microsurgical strategies such as aneurysm trapping and flow-preservation bypass, which increases the operative risks. ${ }^{6}$ The occurrence of any complication $(C D G \geq I)$ was more frequent in patients treated for complex aneurysms than in patients treated for noncomplex aneurysms $(p=0.06)$. The frequency of new neurological deficits was significantly higher in patients who underwent surgery for complex aneurysms versus those who underwent surgery for noncomplex aneurysms $(p=0.007)$. The statistical association between aneurysm complexity and the occurrence of new neurological deficit confirms the predictive value for complications of the definition given for aneurysm complexity. ${ }^{21}$

\section{Advantages and Disadvantages of the CDG}

The CDG provides a simple, reproducible, objective, and applicable therapy-oriented way to classify postsurgical complications-precisely the aim of Clavien et al. ${ }^{13}$
There is no subjective component, such as "minor" or "major" complications, and the grading system enables comparison between case series in different institutions. The CDG has been widely used in other surgical disciplines $^{5,22,23}$ and, thus, facilitates comparisons between surgical specialties. ${ }^{24}$ Furthermore, there is no underreporting of complications due to the registration of any deviation from the standard postsurgical procedure, as a definition of the CDG classification system.

Several studies have shown that the CDG is clinically relevant and associated with patient outcomes and with hospital LOS in spine, orthopedic, and visceral surgery. ${ }^{23-26}$ Nevertheless, it is not evident that the CDG can be used for cranial surgery, where complications can cause severe neurological deficits. Some of the neurological complications do not demand sophisticated invasive treatments and are, therefore, graded low on the CDG scale, although they may be associated with worse patient outcomes.

The same concern was discussed by Bellut et al. ${ }^{24}$ for patients undergoing lumbar spine surgery. Similarly, in a recently published retrospective, observational two-center study on consecutive patients who underwent brain tumor resection, the majority of complications in primary as well as in repeat tumor surgeries were a CDG I or II, thus not requiring invasive treatment. ${ }^{27}$

The CDG classification system has a significant limitation in that the CDG depends only on the therapy that the patient receives for a complication. The impact of complications on dependence in daily activities or the severity of neurological deficits is not included in the CDG system. In the original CDG classification of $2004,{ }^{13}$ the suffix "d," which can be added to any CDG, was used to indicate a persistent (disabling) complication at the time of discharge or follow-up that compromised the patient's health and was directly related to the treatment.

Based on our experience with use of the CDG in neurosurgery, ${ }^{24,27,28}$ the suffix " $d$ " indicates any new neurological deficit after a neurosurgical procedure. This is of importance because some neurological complications (e.g., motor deficit or aphasia) do not demand pharmacological or surgical treatments and are, therefore, given a low CDG, although they may be associated with worse patient outcomes. As seen in Fig. 1B and C, among 9 patients who had a CDG of I, 7 patients experienced new neurological symptoms resulting in higher mRS and NIHSS scores. This finding illustrates the usefulness of affixing " $\mathrm{d}$ " to the CDG to additionally score neurological deficit and avoid underestimating complications.

\section{Limitations of the Study}

The follow-up analysis and CDG validation were limited to 3 months, which could have missed some delayed postoperative complications. However, other published complication studies in neurosurgery also used a similar follow-up period. ${ }^{24,28}$ Second, the treating physician performed the outcome assessment. Third, all analyses were carried out on data from a single reference center. Therefore, the study population is small due to a selected cohort of patients, and the results cannot be generalized to other populations and centers with different decision protocols. Due to the limited number of patients, subgroup analysis 
or multivariate analysis for baseline risk factors was not performed. An external validation or major, multicenter study is required to confirm our findings.

\section{Conclusions}

We documented the applicability of the CDG classification system to neurosurgical patients who received microsurgical treatment of unruptured UIAs. We found a significant correlation between CDG and clinical outcome scales (mRS and NIHSS), as well as the hospital LOS. The CDG "d" is important for scoring neurological deficits and avoiding underestimation of complications. Research on outcomes after neurosurgical treatments is of growing importance to improve patient involvement in decision-making, standard of care, and outcome results. Therefore, the future direction will have to combine the CDG, outcome scales, and neurological deficits to better appraise adverse events.

\section{References}

1. Vlak MH, Algra A, Brandenburg R, Rinkel GJ. Prevalence of unruptured intracranial aneurysms, with emphasis on sex, age, comorbidity, country, and time period: a systematic review and meta-analysis. Lancet Neurol. 2011;10(7):626-636.

2. Brown RD Jr, Broderick JP. Unruptured intracranial aneurysms: epidemiology, natural history, management options, and familial screening. Lancet Neurol. 2014;13(4):393-404.

3. Esposito G, Dias SF, Burkhardt JK, et al. Selection strategy for optimal keyhole approaches for MCA aneurysms: lateral supraorbital versus minipterional craniotomy. World Neurosurg. 2019;122:e349-e357.

4. Etminan N, Rinkel GJ. Unruptured intracranial aneurysms: development, rupture and preventive management. Nat Rev Neurol. 2016;12(12):699-713.

5. Clavien PA, Sanabria JR, Strasberg SM. Proposed classification of complications of surgery with examples of utility in cholecystectomy. Surgery. 1992;111(5):518-526.

6. Esposito G, Fierstra J, Regli L. Distal outflow occlusion with bypass revascularization: last resort measure in managing complex MCA and PICA aneurysms. Acta Neurochir (Wien). 2016;158(8):1523-1531.

7. Esposito G, Durand A, Van Doormaal T, Regli L. Selectivetargeted extra-intracranial bypass surgery in complex middle cerebral artery aneurysms: correctly identifying the recipient artery using indocyanine green videoangiography. Neurosurgery. 2012;71(2 Suppl Operative):ons274-ons285.

8. Jafar JJ, Russell SM, Woo HH. Treatment of giant intracranial aneurysms with saphenous vein extracranial-to-intracranial bypass grafting: indications, operative technique, and results in 29 patients. Neurosurgery. 2002;51(1):138-146.

9. Lawton MT, Hamilton MG, Morcos JJ, Spetzler RF. Revascularization and aneurysm surgery: current techniques, indications, and outcome. Neurosurgery. 1996;38(1):83-94.

10. Esposito G, Regli L. Surgical decision-making for managing complex intracranial aneurysms. Acta Neurochir Suppl. 2014; 119:3-11.

11. Esposito G, Amin-Hanjani S, Regli L. Role of and indications for bypass surgery after Carotid Occlusion Surgery Study (COSS)? Stroke. 2016;47(1):282-290.

12. Esposito G, Fierstra J, Regli L. Partial trapping strategies for managing complex intracranial aneurysms. Acta Neurochir Suppl. 2016;123:73-75.

13. Clavien PA, Barkun J, de Oliveira ML, Vauthey JN, Dindo $\mathrm{D}$, Schulick RD, et al. The Clavien-Dindo classification of surgical complications: five-year experience. Ann Surg. 2009; 250(2):187-196.
14. Dindo D, Demartines N, Clavien PA. Classification of surgical complications: a new proposal with evaluation in a cohort of 6336 patients and results of a survey. Ann Surg. 2004; 240(2):205-213.

15. R Core Team. R: A Language and Environment for Statistical Computing. R Foundation for Statistical Computing; 2020.

16. Dias SF, Jehli E, Regli L, Sarnthein J, Germans MR. Multimodal monitoring strategy is decisive in elective middle cerebral artery aneurysm clipping: a case report. World Neurosurg. 2019;122:43-47.

17. King JT Jr, Berlin JA, Flamm ES. Morbidity and mortality from elective surgery for asymptomatic, unruptured, intracranial aneurysms: a meta-analysis. J Neurosurg. 1994;81(6): 837-842.

18. Krisht AF, Gomez J, Partington S. Outcome of surgical clipping of unruptured aneurysms as it compares with a 10-year nonclipping survival period. Neurosurgery. 2006;58(2):207216.

19. Raaymakers TW, Rinkel GJ, Limburg M, Algra A. Mortality and morbidity of surgery for unruptured intracranial aneurysms: a meta-analysis. Stroke. 1998;29(8):1531-1538.

20. Kotowski M, Naggara O, Darsaut TE, Nolet S, Gevry G, Kouznetsov E, Raymond J. Safety and occlusion rates of surgical treatment of unruptured intracranial aneurysms: a systematic review and meta-analysis of the literature from 1990 to 2011. J Neurol Neurosurg Psychiatry. 2013;84(1):42-48.

21. Staartjes VE, Sebök M, Blum PG, Serra C, Germans MR, Krayenbühl N, et al. Development of machine learning-based preoperative predictive analytics for unruptured intracranial aneurysm surgery: a pilot study. Acta Neurochir (Wien). 2020;162(11):2759-2765.

22. Lee CY, Huang TJ, Li YY, Cheng CC, Wu MH. Comparison of minimal access and traditional anterior spinal surgery in managing infectious spondylitis: a minimum 2-year followup. Spine J. 2014;14(7):1099-1105.

23. Mitropoulos D, Artibani W, Biyani CS, Bjerggaard Jensen J, Roupret M, Truss M. Validation of the Clavien-Dindo Grading System in Urology by the European Association of Urology Guidelines Ad Hoc Panel. Eur Urol Focus. 2018;4(4): 608-613.

24. Bellut D, Burkhardt JK, Schultze D, Ginsberg HJ, Regli L, Sarnthein J. Validating a therapy-oriented complication grading system in lumbar spine surgery: a prospective populationbased study. Sci Rep. 2017;7(1):11752.

25. Garcia-Garcia ML, Martin-Lorenzo JG, Liron-Ruiz R, Torralba-Martinez JA, Garcia-Lopez JA, Aguayo-Albasini JL. Perioperative complications following bariatric surgery according to the clavien-dindo classification. Score validation, literature review and results in a single-centre series. Surg Obes Relat Dis. 2017;13(9):1555-1561.

26. Monteiro E, Sklar MC, Eskander A, de Almeida JR, Shrime M, Gullane P, et al. Assessment of the Clavien-Dindo classification system for complications in head and neck surgery. Laryngoscope. 2014;124(12):2726-2731.

27. Zattra CM, Zhang DY, Broggi M, Velz J, Vasella F, Seggewiss D, et al. Repeated craniotomies for intracranial tumors: is the risk increased? Pooled analysis of two prospective, institutional registries of complications and outcomes. $J$ Neurooncol. 2019;142(1):49-57.

28. Landriel Ibañez FA, Hem S, Ajler P, Vecchi E, Ciraolo C, Baccanelli M, et al. A new classification of complications in neurosurgery. World Neurosurg. 2011;75(5-6):709-715, 604611.

\section{Disclosures}

Dr. Regli: speakers bureau for B. Braun. 


\section{Author Contributions}

Conception and design: Sebök, Sarnthein, Regli, Esposito. Acquisition of data: Sebök, Blum. Analysis and interpretation of data: Sebök, Blum, Esposito. Drafting the article: Sebök, Blum. Critically revising the article: all authors. Reviewed submitted version of manuscript: Sebök, Blum, Sarnthein, Fierstra, Germans, Serra, Krayenbühl, Regli. Approved the final version of the manuscript on behalf of all authors: Sebök. Statistical analysis: Sebök.

Administrative/technical/material support: Esposito. Study supervision: Sarnthein, Regli, Esposito.

\section{Correspondence}

Martina Sebök: University Hospital Zurich, Switzerland. martina. seboek@usz.ch. 